\title{
An amenable equivalence relation is generated by a single transformation
}

\author{
A. CONNES, J. FELDMAN AND B. WEISS \\ Institut des Hautes Etudes Scientifiques, 35 route de Chartres, 91440 \\ Bures-sur-Yvette, France; Department of Mathematics, University of California \\ at Berkeley, California, USA; Hebrew University of Jerusalem, Israel
}

(Received 25 May 1981 and revised 1 September 1981)

Abstract. We prove that for any amenable non-singular countable equivalence relation $R \subset X \times X$, there exists a non-singular transformation $T$ of $X$ such that, up to a null set:

$$
R=\left\{\left(x, T^{n} x\right) ; x \in X, n \in \mathbb{Z}\right\} .
$$

It follo:vs that any two Cartan subalgebras of a hyperfinite factor are conjugate by an automorphism.

\section{Statement of tile results}

The main result of this paper is that, for any amenable non-singular (n.s.) countable equivalence relation $R \subset X \times X$, there exists a non-singular transformation $T$ of $X$ such that, up to a null set,

$$
R=\left\{\left(x, T^{n} x\right), x \in X, n \in \mathbb{Z}\right\} .
$$

Roughly speaking, the equivalence relation $R$ is amenable if and only if one can define a measurable family of positive linear functionals $p_{x}$ on $l^{\infty}\left(R^{x}\right)$ (where $\left.R^{x}=R \cap(\{x\} \times X)\right)$ with

$$
p_{x}(1)=1 \text {, }
$$

which is invariant in the sense that, for $(x, y) \in R, p_{y}$ is the image of $p_{x}$ by the left translation: $(x, z) \in R^{x} \mapsto(y, z) \in R^{y}$. If $R$ is given by the (not necessarily free) action of an amenable group, then it is straightforward to see that it is amenable. However, the equivalence relation of a non-singular action of a discrete group $\Gamma$ can be amenable even though the group is not amenable. For instance, R. Zimmer [32] showed that, if $\Gamma$ is a discrete subgroup of a Lie group, then the action of $\Gamma$ on $G / P$ where $P$ is solvable, is amenable. In particular, $\Gamma$ acting on the Furstenberg boundary $B(G)$ is amenable. As a rule it is easier to check the amenability of an equivalence relation than to generate it by a single transformation. For instance, let $\Gamma \subset S L(2, \mathbb{R})$ be discrete. Then it will act amenably on $P_{1}(\mathbb{R})$. However, to generate the corresponding equivalence relation by a single transformation is delicate even in the simplest case when $\Gamma=\operatorname{SL}(2, \mathbb{Z})$.

Putting together the main result of our paper and the classification of $W$. Krieger [18] of a non-singular transformation up to weak equivalence, we obtain that the 
amenable countable equivalence relations are classified by non-singular flows. The amenability of a countable non-singular equivalence relation follows directly from the amenability of the associated von Neumann algebra (via the so-called 'group measure space construction').

This, together with the above-mentioned result, shows that a given amenable von Neumann algebra $M$ arises from at most one countable n.s. equivalence relation, which implies the uniqueness, up to an automorphism of $M$, of a Cartan subalgebra of $M$. In the case $\mathrm{II}_{1}$, a Cartan subalgebra is simply a maximal abelian subalgebra $\mathscr{A}$ of $M$ whose normalizer generates $M$ (as a von Neumann algebra). In the general case one has to assume further that $\mathscr{A}$ is discretely imbedded in $M$, i.e. that it is contained in the centralizer of a normal state (or equivalently is the range of a normal conditional expectation).

\section{Background}

Since the papers of E. Hopf [15] and the first paper 'On rings of operators' [21] of Murray and von Neumann, and their construction of factors from non-singular actions of discrete groups on Lebesgue measure spaces, there has been a fruitful interplay between the theory of von Neumann algebras and that part of ergodic theory dealing with orbit equivalence.

In [22] Murray and von Neumann showed uniqueness, up to isomorphism, of factors of type $\mathrm{II}_{1}$ which are well approximated by finite dimensional subalgebras; they called this factor 'approximately finite'. Since factors of type $\mathrm{II}_{1}$ were also called finite, the terminology was then modified, the above factor being called hyperfinite. In [22] Murray and von Neumann showed that, if $\Gamma$ is a locally finite group, ergodic and probability preserving, then the factor obtained by the group measure space construction is hyperfinite. They also stated that the condition ' $\Gamma$ is locally finite' can be replaced by ' $\Gamma$ is abelian' (cf. [22] lemma 5.2.3): 'The proof of this lemma is somewhat complicated. It requires some rather deep results on the decomposition of mappings of measurable sets which will be published elsewhere. We shall not pursue this matter further on this occasion.' In his two papers [9], [10] H. Dye established the hyperfiniteness of the above factors with $\Gamma$ abelian, and also more generally for $\Gamma$ of polynomial growth. In fact, he proved much more, showing that the isomorphism of the factors occurs at a more basic and purely measure theoretic level, as an orbit equivalence of the actions. Several years later Belinskaya and A. M. Veršik [1] and, independently, W. Krieger discovered the connection between the work of $\mathrm{H}$. Dye and the classical so-called Rohlin lemma of ergodic theory. At this point we shall describe the history of the Rohlin lemma in ergodic theory, which is one of the strands that led to the result of this paper. Afterwards, we shall elaborate on the notion of amenability, the other strand leading to our result.

In [24] Rohlin proved that an arbitrary aperiodic probability preserving transformation $T$ can be well approximated by periodic transformations. For this purpose he proved the lemma which now bears his name, which states that, given $\varepsilon>0$ and $n \in \mathbb{N}$, there exisis a set $B$ so that the $T^{i} B$ are pairwise disjoint for $0 \leq i<n$, and 
cover the whole space up to $\varepsilon$. This lemma was extended to the case of a general non-singular aperiodic transformation by $\mathrm{C}$. Linderholm (see [16] and [3]). At the same time the Rohlin lemma turned out to be an extremely useful tool in the ergodic theory of a single transformation, i.e. actions of the group $\mathbb{Z}$. For the groups $\mathbb{Z}^{\nu}, \nu \in \mathbb{N}$, the analogue of this lemma was proved in the probability preserving case by J. P. Conze [8] and I. Katznelson and B. Weiss [17], and in the non-singular case by J. Feldman and D. Lind [11].

If the 'Rohlin lemma' holds for a non-singular action of a discrete group $\Gamma$, then it follows easily that this action is orbit equivalent to $a \mathbb{Z}$ action. This latter property was proved directly for solvable $\Gamma$ by $A$. Connes and W. Krieger in [7]. The 'Rohlin lemma' itself was proven for solvable $\Gamma$ by $D$. Ornstein and B. Weiss in [23]. $H$. Dye had conjectured in [10] that an arbitrary action of an amenable $\Gamma$ is orbit equivalent to a $\mathbb{Z}$ action; this conjecture was proved by $\mathrm{D}$. Ornstein and $\mathrm{B}$. Weiss in [24]. The fact that an arbitrary action of an amenable $\Gamma$ gives rise (via the group measure space construction) to a hyperfinite von Neumann algebra had already been established by purely operator theoretic methods by A. Connes in [5]. The crucial property that the von Neumann algebra inherits from the amenability of $\Gamma$ is the property $\mathrm{P}$, introduced by J. Schwartz in 1962 [26], which can be reformulated as the existence of a projection $E$ of norm one from all bounded operators onto the algebra. In fact, for a free and probability preserving action of a discrete group $\Gamma$, the associated algebra has property $P$ if and only if $\Gamma$ is amenable, as was already observed by V. Golodets in [14]. In the non-singular case, however, non-amenable groups can give rise to an algebra with property $P$. The fact that the associated algebra has property $\mathbf{P}$ has a purely measure theoretical formulation, which was investigated in detail by R. Zimmer in [30], [31], and which is the notion of amenability for equivalence relations that we defined above. The present paper closes this circle of ideas by establishing directly that an amenable non-singular countable equivalence relation can be generated by a single transformation.

\section{Discrete measured equivalence relations}

Let $X$ be a standard Borel space and $\mathrm{R}$ a Borel subset of $X \times X$ which is an equivalence relation. We say that $R$ is discrete if each $R$ equivalence class is countable. A measure $\mu$ on $X$ is said to be quasi-invariant for $R$ if, for every $\mu$-null Borel set $A \subset X$, the saturation of $A, R(A)=\{x \in X, \exists y \in A,(x, y) \in R\}$, is $\mu$-null.

By an isomorphism of discrete measured equivalence relations, from $R_{1}$ to $R_{2}$, we mean a measure class preserving bijection $\sigma$ from $X_{1}$ to $X_{2}$ such that

$$
\sigma \times \sigma\left(R_{1}\right)=R_{2} .
$$

By a partial transformation $\phi$ of $X$ we mean a pair of measurable subsets Dom $\phi$, $\operatorname{Im} \phi \subset X$ and a measurable bijection $\phi$ from $\operatorname{Dom} \phi$ to $\operatorname{Im} \phi$. We identify $\phi$ with its graph,

and write $\phi \subset R$ if one has

$$
\{(x, \phi(x)), x \in \operatorname{Dom} \phi\},
$$

$$
(x, \phi(x)) \in R \quad \text { for all } x \in \operatorname{Dom} \phi .
$$


If $\phi \subset R$ then the image by $\phi$ of a Borel $\mu$-null set is $\mu$-null, i.e. $\phi$ is non-singular.

We shall consider $R$ as a groupoid, with units $R^{(0)}=X$, range and source maps

$$
r(y, x)=y, \quad s(y, x)=x
$$

and composition

$$
(z, y) \cdot(y, x)=(z, x) .
$$

There is a Borel homomorphism $\delta$,

$$
\delta((x, y) \cdot(y, z))=\delta(x, y) \delta(y, z), \quad \forall x, y, z,
$$

from $R$ to ]0, $+\infty[$, called the module of $\mu$ such that, for every partial transformation $\phi \in R$, one has

$$
d \mu(\phi(x)) / d \mu(x)=\delta(\phi(x), x)
$$

a.e. on Dom $\phi$.

One endows $R$ with the measure

where $\nu^{x}$ is the counting measure on

$$
m=\int \nu^{x} d \mu(x)
$$

$$
R^{x}=\{(x, y), y \sim x\},
$$

or with the equivalent measure $m^{-1}$,

$$
d m^{-1}(x, y)=\delta^{-1}(x, y) d m(x, y)
$$

which is the image of $m$ by

$$
(x, y) \in R \mapsto(y, x)=(x, y)^{-1} .
$$

Definition 1. (1) We say that the discrete measured equivalence relation $R$ is of type I (or smooth) when the quotient Borel space is, up to a null set, a standard space.

(2) We say that the discrete measured equivalence relation $R$ is hyperfinite (or approximately smooth) when it is, up to a null set, a countable increasing union of type I equivalence relations.

Let us recall some well-known facts:

$R$ is of type $\mathrm{I}$ if and only if $X$ is a countable union of subsets $X_{n}$ on which $R$ is trivial. So, if $R$ is of type I and $R_{1} \subset R$, then $R_{1}$ is of type I.

$R$ is hyperfinite if and only if there is a measurable transformation $T$, such that (up to an $m$-null set)

$$
R=\bigcup_{n \in \mathbf{Z}} \text { Graph } T^{n}
$$

If $R$ is hyperfinite and $R_{1} \subset R$, then $R_{1}$ is hyperfinite, as follows from the definition and the above remark on type I equivalence relations.

\section{Finite subequivalence relations}

Let $R$ be an equivalence relation as above. By a finite subequivalence relation (f.s.r.), we mean a measurable subset $T$ of $R$ which is the graph of an equivalence relation with finite equivalence classes on the subset

$$
T^{(0)}=T \cap R^{(0)}=\{x \in X,(x, x) \in T\}
$$


of $X$. Given f.s.r. $T \subset T^{\prime}$ we say that $T^{\prime}$ is an extension of $T$ when it is the union of $T$ and of a disjoint f.s.r. $T^{\prime \prime}$. Equivalently,

$$
(y, x) \in T^{\prime}, \quad x \text { or } y \in T^{(0)} \Rightarrow(y, x) \in T .
$$

If $\left(T_{n}\right)$ is a sequence of f.s.r. and $T_{n+1}$ extends $T_{n}$ for all $n$, then

$$
T=\bigcup_{1}^{\infty} T_{n}
$$

is also a f.s.r.

Let $T$ be a f.s.r. We shall call a fibre any equivalence class of $T$. We let $Q$ be the space of fibres and $\mu_{T}$ the image on $Q$ of the restriction of $\mu$ to $T^{(0)} ; Q$ is, of course, a standard Borel space, up to a null set. For each fibre $F$ one has the probability measure $\nu_{F}$ on $F$ which is the conditional measure of the restriction of $\mu$ to $T^{(0)}$ :

$$
\nu_{F}(A)=\frac{\sum_{x \in A} \delta^{-1}(y, x)}{\sum_{x \in F} \delta^{-1}(y, x)}, \quad \forall y \in F .
$$

We describe the disintegration of $\mu / T^{(0)}$ by a lemma.

LEMMA 2. For any measurable subset $A$ of $T^{(0)}$ one has

$$
\mu(A)=\int_{Q} \nu_{F}(A) d \mu_{T}(F) .
$$

\section{Bounded subsets of $R$}

Let $K$ be a measurable subset of $R$. We shall say that $K$ is bounded if $\operatorname{Sup}_{x}\left|K_{x}\right|$, $\operatorname{Sup}_{y}\left|K^{y}\right|, \operatorname{Sup}_{K}|\log \delta|$ are all finite.

LEMMA 3. (a) $R$ is a countable union of bounded measurable subsets.

(b) Any bounded subset $K$ of $R$ is equal to a finite union of graphs of partial transformations $\phi_{i}$ with bounded Radon-Nikodym derivatives.

Proof. Even though the lemma can be easily deduced from [12] we shall sketch a proof for the reader's convenience.

(a) The equivalence relation $R$ is a countable union of graphs of Borel transformations $\phi_{n}$ (theorem 1 of [12]). The conclusion follows by restricting $\phi_{n}$ to Borel subsets $E_{n, m}$ of $X$,

$$
\bigcup_{m} E_{n, m}=X
$$

on which $\left|\log \left(d \mu \circ \phi_{n} / d \mu\right)\right|$ is bounded.

(b) We can assume that $R$ is identical as a Borel space with $[0,1]$. Then, with

$$
n=\operatorname{Sup}_{y}\left|K^{y}\right|
$$

define the partial maps $\psi_{i}, i=1, \ldots, n$, by

$$
\psi_{i}(y)=s\left(\gamma_{i}\right)
$$


where $\gamma_{i}$ is the $i$ th element of $K^{y}$ (in the ordering of $[0,1]$ ). Clearly, $\psi_{i}$ is measurable,

$$
\text { Graph } \psi_{i} \subset R \text {, }
$$

and

$$
K=\bigcup \text { Graph } \psi_{i} .
$$

$\psi_{i}$ can fail to be injective, but $\psi_{i}$ is at most $m$ to 1 , where

$$
m=\operatorname{Sup}_{x}\left|K_{x}\right| .
$$

Using again the ordering of $[0,1]$, one writes

$$
\operatorname{Dom} \psi_{i}=\bigcup_{j=1}^{m} \operatorname{Dom} \psi_{i, j}
$$

with $\psi_{i j}$ injective,

$$
\psi_{i j}(y)=\psi_{i}(y), \quad \forall y \in \operatorname{Dom} \psi_{i j} .
$$

Then

$$
\boldsymbol{K}=\bigcup \operatorname{Graph}\left(\psi_{i j}\right) .
$$

The next lemma, though very easy, will be crucial in the proof. It expresses the 'local triviality' of the equivalence relation $R$.

LEMMA 4. Let $K$ be a bounded subset of $R$. Then, for every $\Omega \subset X, \mu(\Omega)>0$, there exists $\Omega^{\prime} \subset \Omega, \mu\left(\Omega^{\prime}\right)>0$ with $s(x \cdot K)$ disjoint from $s(y \cdot K)$ for all $x, y \in \Omega^{\prime}, x \neq y$. Proof. First let $\phi$ be a partial transformation of $X, \Omega \subset X, \mu(\Omega)>0$. Then there exists $\Omega^{\prime} \subset \Omega, \mu\left(\Omega^{\prime}\right)>0$ such that one of the following three conditions holds:

(a) $\Omega^{\prime} \cap \operatorname{Dom} \phi=\varnothing$;

( $\beta$ ) $\Omega^{\prime} \subset \operatorname{Dom} \phi$ and $\phi\left(\Omega^{\prime}\right) \cap \Omega^{\prime}=\varnothing$;

$(\gamma) \Omega^{\prime} \subset \operatorname{Dom} \phi$ and $\phi(x)=x, \forall x \in \Omega^{\prime}$.

Now let $\phi_{i}$ be as in lemma $3(b)$. Applying the above procedure to the $\phi_{i}^{-1} \circ \phi_{i}$, one obtains $\Omega^{\prime} \subset \Omega, \mu\left(\Omega^{\prime}\right)>0$, such that, for all $(i, j)$, one has one of the three following conditions:

$$
\begin{aligned}
& \left(\alpha^{\prime}\right) \Omega^{\prime} \cap \text { Dom } \phi_{i}^{-1} \circ \phi_{i}=\varnothing ; \\
& \left(\beta^{\prime}\right) \Omega^{\prime} \subset \operatorname{Dom} \phi_{i}^{-1} \circ \phi_{i} \text { and } \phi_{i}^{-1} \circ \phi_{j}\left(\Omega^{\prime}\right) \cap \Omega^{\prime}=\varnothing ; \\
& \left(\gamma^{\prime}\right) \Omega^{\prime} \subset \operatorname{Dom} \phi_{i}^{-1} \circ \phi_{j} \text { and } \phi_{i}(x)=\phi_{j}(x), \forall x \in \Omega^{\prime} .
\end{aligned}
$$

For $x, y \in \Omega^{\prime}, x \neq y$ and $\phi_{i}(x)=\phi_{j}(y)$ one obtains $y \in \operatorname{Dom} \phi_{i}^{-1} \circ \phi_{j}$ and hence a contradiction with $\left(\alpha^{\prime}\right),\left(\beta^{\prime}\right)$ or $\left(\gamma^{\prime}\right)$.

\section{Amenability of $R$}

Let $R, \mu$ and $m$ be as above. For any function $f$ on $R$ and partial transformation $\phi$, one defines a function $f^{\phi}$ (resp. $f_{\phi}$ ) by

$$
f^{\phi}(y, x)=0 \quad \text { if } y \notin \operatorname{Im} \phi
$$

(resp. $f_{\phi}=0$ if $\left.x \notin \operatorname{Im} \phi\right)$;

$$
f^{\phi}(y, x)=f\left(\phi^{-1}(y), x\right) \quad \text { if } y \in \operatorname{Im} \phi
$$

(resp. $f_{\phi}(y, x)=f\left(y, \phi^{-1}(x)\right)$ if $\left.x \in \operatorname{Im} \phi\right)$. For functions $f$ on $X, f_{\phi}=f^{\phi}$ is defined as $f \circ \phi^{-1}$ on $\operatorname{Im} \phi$ and 0 on $(\operatorname{Im} \phi)^{c}$. 
We now define the notion of a left (resp. right) invariant mean on $R$. As we shall see, the existence of such a mean is a simple translation of the amenability of the associated von Neumann algebra and is, of course, equivalent to the amenability of $R$ in the sense of R. Zimmer [27].

To motivate the definition, assume one is given for each $x \in X$ a state $p_{x}$ on $l^{\infty}\left(R^{x}\right)$ in such a way that, for $\gamma \in R, \gamma: x \rightarrow y$, one has

$$
\gamma p_{x}=p_{y}
$$

(i.e. $p_{y}(f)=p_{x}(f(\gamma \cdot))$ and that, for $f \in L^{\infty}(R, m)$, the function on $X, x \mapsto p_{x}(f)$, is $\mu$-measurable. Then the map $P$ from $L^{\infty}(R, m)$ to $L^{\infty}(X, \mu)$ such that

$$
(P f)(x)=p_{x}(f)
$$

is a left invariant mean on $R$ in the following sense.

Definition 5. A left (resp. right) invariant mean on $R$ is a positive map $P, P(1)=1$, from $L^{\infty}(R, m)$ to $L^{\infty}(X, \mu)$ such that for any partial transformation $\phi \subset R$ one has

$$
P\left(f^{\phi}\right)=(P f)^{\phi} \quad\left(\text { resp. } P\left(f_{\phi}\right)=(P f)_{\phi}\right) .
$$

In the previous situation, one has

$$
P\left(f^{\phi}\right)(x)=p_{x}\left(f^{\phi}\right)=0 \quad \text { if } x \notin \operatorname{Im} \phi
$$

(then $f^{\phi}(x, a)=0$ for all $a$ ) and if $x=\phi(b)$ one has with

$$
\begin{gathered}
\gamma=(b, x), \\
f^{\phi}(x, a)=f(b, a)=f(\gamma \cdot(x, a))
\end{gathered}
$$

so

$$
p_{x}\left(f^{\phi}\right)=p_{b}(f) .
$$

It turns out, but we shall not need it, that the existence of a left (resp. right) invariant mean on $R$ is equivalent to the existence of a left (resp. right) invariant family $\left(p_{x}\right)$ as above. The main use of this fact is that the conditions satisfied by the family $\left(p_{x}\right)$ are easier to handle than those for $P$.

Definition 6. $R$ is amenable if and only if it possesses a left (or equivalently a right) invariant mean.

In the rest of this section we connect this notion with the amenability [5], [6] of the associated von Neumann algebra. For the application to operator algebras we need the construction of the projective regular representations of $R$ [12].

Let $c$ be a normalized multiplier for $R$, i.e. $c$ is a Borel map from

$$
R^{(2)}=\left\{(z, y, x) \in X^{3}, x \sim y \sim z(R)\right\}
$$

to

$$
U(1)=\{z \in \mathbb{C},|z|=1\}
$$

such that, for all $x \sim y \sim z \sim t(R)$, one has

$$
c(t, z, x) c(z, y, x)=c(t, y, x) c(t, z, y) .
$$

For each Borel function $a$ on $R$ and each $x \in X$, consider the matrix $c(y, t, x) a(y, t)$, where $y$ and $t$ vary arbitrarily in the (countable) equivalence class of $x$. We let $\|a\|_{\infty}$ 
be the essential supremum of the operator norms of the above matrices, so

$$
\|a\|_{\infty} \in[0,+\infty]
$$

(Note that if $x^{\prime} \sim x$ one has

$$
c\left(y, t, x^{\prime}\right) a(y, t)=\alpha(y) c(y, t, x) a(y, t) \overline{\alpha(t)},
$$

where $\alpha(y)=c\left(y, x, x^{\prime}\right)$ is a complex number of modulus one. Thus the operator norm of the matrix $c(y, t, x) a(y, t)$ is independent of the choice of $x$ in its $R$ equivalence class. In particular, if $\mu$ is ergodic, the above essential supremum is also an essential value.) Let $M$ be the vector space of all Borel functions $a$ on $R$ such that $\|a\|_{\infty}<\infty$ modulo the subspace $\left\{a,\|a\|_{\infty}=0\right\}$ of those which are zero almost everywhere. Endowed with the pointwise product and ${ }^{*}$ operation (which do not depend on the choice of $x$ in its $R$ equivalence class) of the matrices $(c(y, t, x) a(y, t))_{y, t \sim x}, M$ becomes an abstract von Neumann algebra. It can also be defined as the von Neumann algebra in $L^{2}(R, m)$ generated by the left regular representation $\lambda$ of $R$ defined as follows. To each $\phi \subset R$ one associates a partial isometry $\lambda(\phi)$ such that

$$
(\lambda(\phi) f)(y, x)=c\left(y, \phi^{-1}(y), x\right) f^{\phi}(y, x), \quad \forall f \in L^{2}(R, m) .
$$

More generally, the bounded operator

$$
(\lambda(a) f)(y, x)=\sum a(y, t) f(t, x) c(y, t, x), \quad \forall f \in L^{2}(R, m),
$$

corresponds to $a \in M$. One defines in the same way the right regular representation $\rho$, using the natural isometry from $L^{2}\left(R, m^{-1}\right)$ to $L^{2}(R, m) ; \rho(\phi)$ acts in $L^{2}(R, m)$ in the following way:

$$
(\rho(\phi) f)(y, x)=c\left(y, \phi^{-1}(x), x\right) f_{\phi}(y, x) .
$$

The von Neumann algebras $\lambda(R), \rho(R)$ generated respectively by the $\lambda(\phi), \rho(\phi), \phi \subset R$, are commutants of each other. In $\lambda(R)$ the operators $\lambda(A), A$ a measurable subset of $X$, are exactly the projections of a maximal abelian subalgebra $\lambda(X)$ of $\lambda(R)$.

Up to algebraic isomorphism, the pairs $(M, C)$ of a von Neumann algebra and a maximal abelian subalgebra that one obtains in this way are characterized by two properties. (They are called Cartan subalgebras in [12]; see also [28].)

(1) $C$ is discretely imbedded in $M$; i.e. there exists a normal projection of norm one of $M$ on $C$.

(2) $C$ is regular; i.e. its normalizer generates $M$ as a von Neumann algebra.

In the abeve spatial realization $\zeta^{\infty}(R, m)$ acts on $L^{2}(R, m)$ by multiplication and one has the following:

$$
\begin{aligned}
& \lambda(\phi) f \lambda(\phi)^{*}=f^{\phi}, \\
& \rho(\phi) f \rho(\phi)^{*}=f_{\phi},
\end{aligned}
$$

for all $f \in L^{\infty}(R, m)$ and partial transformations $\phi \subset R$.

PROPOSITION 7. Let $E$ be a projection of norm one from the algebra $\mathscr{L}\left(L^{2}(R, m)\right)$ of all operators in $L^{2}(R, m)$ on $\lambda(R)$ (resp. $\rho(R)$ ). Then the restriction of $E$ to $L^{\infty}(R, m)$ is a left (resp. right) invariant mean on $R$. 
Proof. One has

$$
E(a T b)=a E(T) b
$$

for $a, b \in \lambda(R)$ and $T \in \mathscr{L}\left(L^{2}(R, m)\right)$. This shows that

$$
E(f) \in \lambda(R) \cap \lambda(X)^{\prime}=\lambda(X)
$$

for $f \in L^{\infty}(R, m)$ and that

$$
E\left(f^{\phi}\right)=E\left(\lambda(\phi) f \lambda(\phi)^{*}\right)=\lambda(\phi) E(f) \lambda(\phi)^{*}=E(f)^{\phi} .
$$

Conversely, if the discrete measured equivalence relation $R$ is amenable, the associated von Neumann algebra $\lambda(R)$ is the range of a projection of norm one from $\mathscr{L}\left(L^{2}(R, m)\right)$. Since we shall not need this converse, we shall content ourselves with the following sketch of the proof, leaving details to the reader. In particular, we ignore the cocycle $c$; i.e. we assume that

$$
c(x, y, x)=1, \quad \forall x, y, z \in X .
$$

This allows one to describe $\lambda(R)$ as the von Neumann algebra of random operators $\left(T_{l}\right)_{l \in \Omega}$, where $\Omega$ is the space of $R$-equivalence classes. For each $l, T_{i}$ is a bounded operator in the Hilbert space $l^{2}(l)$. One only considers bounded measurable random operators. The measurability of $T$ means that, if $\left(\varepsilon_{x}\right)_{x \in l}$ is the canonical basis of $l^{2}(l)$, the matrix coefficients

$$
a(y, x)=\left\langle T_{l} \varepsilon_{x}, \varepsilon_{y}\right\rangle
$$

define a measurable function $a$ on $R$. The boundedness means that

$$
\|T\|_{\infty}=\text { Ess Sup }\left\|T_{l}\right\|
$$

is finite. Finally, one neglects $T$ if $\|T\|_{\infty}=0$, i.e. if $T_{l}=0$ a.e. Now the amenability of $\boldsymbol{R}$ means that one can associate to each $l \in \Omega$ a positive linear functional $p_{l}$ on $l^{\infty}(l), p_{l}(1)=1$, in a measurable manner, i.e. in such a way that, for any $f \in L^{\infty}(R, m)$, the function on $X, x \mapsto p_{\text {class of } x}(f)$, is $\mu$-measurable. Let $N$ be the von Neumann algebra in $L^{2}(R, m)$ of operators which are diagonal in the decomposition of $L^{2}(R, m)$ as the direct integral

$$
\int_{\oplus} l^{2}\left(R^{x}\right) d \mu(x)
$$

We have

$$
\lambda(R) \subset N .
$$

Since $N$ is of type I, to show that $\lambda(R)$ is the range of a projection of norm one from $\mathscr{L}\left(L^{2}(R, m)\right)$ it is enough to construct one from $N$. An arbitrary element $T$ of $N$ is a bounded measurable family $\left(T_{x}\right)_{x \in X}$, where $T_{x}$ acts in $l^{2}$ (class of $x$ ). Using the above $p$, define, for $l \in \Omega, \xi, \eta \in l^{2}(l)$, the random operator $p(T)$ by putting $\left\langle p(T)_{l} \xi, \eta\right\rangle$ equal to $p_{l}$ of the function $x \mapsto\left\langle T_{x} \xi, \eta\right\rangle$. It is then immediate that $p$ is a projection of norm one from $N$ to $\lambda(R)$.

\section{A Følner condition}

The following Følner type condition is equivalent to amenability. We shall assume that the discrete measured equivalence relation $R$ is amenable and prove: 
LEMMA 8. For any bounded measurable subset $K$ of $R$ and any $\varepsilon>0$, there exists a f.s.r. T such that

$$
m\left\{\gamma \in K, s(\gamma) \in T^{(0)} \text {, or } r(\gamma) \in T^{(0)}, \gamma \notin T\right\}<\varepsilon \mu\left(T^{(0)}\right) \neq 0 .
$$

Proof. Let $\phi_{i}, i=1, \ldots, n$, be partial transformations,

$$
\text { Graph } \phi_{i} \subset R \text {, }
$$

with

$$
|\log \delta| \leq C
$$

on $\bigcup_{i}$ Graph $\phi_{i}$ and

$$
K \subset \bigcup_{i} \operatorname{Graph} \phi_{i}
$$

as in lemma 3. Let $P$ be a right invariant mean on $R$ and $L=\mu \cdot P$ be the corresponding state on $L^{\infty}(R, m)$.

For each $i$, let

$$
\beta_{i}(x)= \begin{cases}\delta\left(\phi_{i}^{-1}(x), x\right) & \text { if } x \in \operatorname{Im} \phi_{i} \\ 0 & \text { otherwise }\end{cases}
$$

and

$$
\alpha_{i}(x)= \begin{cases}1 & \text { if } x \in \text { Dorn } \phi_{i} \\ 0 & \text { otherwise }\end{cases}
$$

For $f \in L^{\infty}(R, m)$ define $T_{i} f$ by

$$
\left(T_{i} f\right)(y, x)=\beta_{i}(x) f\left(y, \phi_{i}^{-1}(x)\right)
$$

By hypothesis, we have

$$
P\left(T_{i} f\right)(x)=\beta_{i}(x)(P f)\left(\phi_{i}^{-1}(x)\right) .
$$

For any $g \in L^{\infty}(X, \mu)$ the equality

$$
\int \beta_{i}(x) g\left(\phi_{i}^{-1}(x)\right) d \mu(x)=\int \alpha_{i}(x) g(x) d \mu(x)
$$

shows that

$$
L\left(T_{i} f\right)=L\left(\alpha_{i} f\right), \quad i=1, \ldots, n, \quad \forall f \in L^{\infty}(R, m) .
$$

Let $L^{\infty}(R, m)_{*} \subset L^{\infty}(R, m)^{*}$ be the space of normal linear functionals on $L^{\infty}(R, m)$. In the Banach space product of $n$ copies of $L^{\infty}(R, m)_{*}$ let

$$
Q=\left\{\left(l \cdot T_{i}-l \cdot \alpha_{i}\right)_{i=1, \ldots, n}, \text { where } l \text { is a normal state on } L^{\infty}(R, m)\right\} .
$$

Then $Q$ is convex, and its $\sigma\left(L_{*}^{\infty}, L^{\infty}\right)$ closure in $L_{*}^{\infty}$ contains 0 , because $L$ is in the $\sigma\left(L^{\infty *}, L^{\infty}\right)$ closure of normal states. Thus, since $L^{\infty}$ is the dual of $L_{*}^{\infty}$, the norm closure of $Q$ contains 0 .

Let $\varepsilon>0, l \in L_{*}^{\infty+}$ be such that

$$
\sum_{i=1}^{n}\left\|l \cdot T_{i}-l \cdot \alpha_{i}\right\|<\varepsilon\|l\|_{1}
$$


We identify $L_{*}^{\infty}(R, m)$ with $L^{1}\left(R, m^{-1}\right)$, and let $l$ be the corresponding function on $R$. We can assume that $\operatorname{Supp} l$ is bounded,

$$
\sum_{i=1}^{n}\left|\int\left(T_{i} f\right) l d m^{-1}-\int \alpha_{i} f l d m^{-1}\right|<\varepsilon\|f\|_{\infty}, \quad \forall f \in L^{\infty}
$$

One has

$$
\begin{aligned}
\int\left(T_{i} f\right) l d m^{-1} & =\int \beta_{i}(x) f\left(y, \phi_{i}^{-1}(x)\right) l(y, x) \delta^{-1}(y, x) d m(y, x) \\
& =\int \beta_{i}\left(\phi_{i}(x)\right) f(y, x) l\left(y, \phi_{i}(x)\right) \delta^{-1}\left(y, \phi_{i}(x)\right) d m(y, x),
\end{aligned}
$$

since

$$
d m\left(y, \phi_{i}(x)\right)=d m(y, x) \text { for } x \in \operatorname{Dom} \phi_{i}
$$

Now

$$
\begin{gathered}
\beta_{i}\left(\phi_{i}(x)\right) \delta^{-1}\left(y, \phi_{i}(x)\right)=\delta^{-1}(y, x) \alpha_{i}(x), \\
\int\left(T_{i} f\right) l d m^{-1}=\int f(y, x) l\left(y, \phi_{i}(x)\right) \alpha_{i}(x) d m^{-1}(y, x),
\end{gathered}
$$

and the above inequality is equivalent to

$$
\sum_{i=1}^{n} \int\left|l\left(y, \phi_{i}(x)\right)-l(y, x)\right| \alpha_{i}(x) d m^{-1}(y, x) \leq \varepsilon
$$

Let $E_{a}$ be the characteristic function of $\left[a,+\infty\left[, a \geq 0\right.\right.$. Then for $t, t^{\prime} \geq 0$,

$$
t=\int_{0}^{\infty} E_{a}(t) d t, \quad\left|t-t^{\prime}\right|=\int_{0}^{\infty}\left|E_{a}(t)-E_{a}\left(t^{\prime}\right)\right| d a,
$$

so that by Fubini's theorem one has, with $l_{a}=E_{a} \cdot l$,

$$
\sum_{i} \iint\left|l_{a}\left(y, \phi_{i}(x)\right)-l_{a}(y, x)\right| \alpha_{i}(x) d m^{-1}(y, x) d a<\varepsilon \iint l_{a} d m^{-1} d a .
$$

So let $a>0$ be such that

$$
\sum_{i} \int\left|l_{a}\left(y, \phi_{i}(x)\right)-l_{a}(y, x)\right| \alpha_{i}(x) d m^{-1}(y, x)<\varepsilon \int l_{a} d m^{-1} \neq 0 .
$$

Recalling that

$$
d m^{-1}(y, x)=\delta^{-1}(y, x) d \mu(y),
$$

we obtain

$\sum_{i} \int_{X} \sum_{x}\left|l_{a}\left(y, \phi_{i}(x)\right)-l_{a}(y, x)\right| \alpha_{i}(x) \delta^{-1}(y, x) d \mu(y)<\varepsilon \int_{X}\left(\sum_{x} l_{a}(y, x)\right) \delta^{-1}(y, x) d \mu(y)$.

Thus the set $\Omega$ of $y \in X$ with

$$
\sum_{i} \sum_{x \in \operatorname{Dom} \phi_{i}}\left|l_{a}\left(y, \phi_{i}(x)\right)-l_{a}(y, x)\right| \delta^{-1}(y, x)<\varepsilon \sum_{x} l_{a}(y, x) \delta^{-1}(y, x)
$$

is non-negligible.

Using lemma 4 , let $\Omega^{\prime} \subset \Omega$ be a non-negligible subset such that, for $y_{1}, y_{2} \in \Omega^{\prime}$, $y_{1} \neq y_{2}, s\left(y_{1} \cdot K_{1}\right)$ is disjoint from $s\left(y_{2} \cdot K_{1}\right)$ with $K_{1}=K^{-1} \cup K^{\prime}$ and $l$ (and also $l_{a}$ ) equal to 0 in $K^{\prime c}$. 
For each $y \in \Omega^{\prime}$ consider

$$
F_{y}=\left\{x \sim y, l_{a}(y, x)=1\right\} .
$$

Then $F_{y} \subset s\left(y \cdot K^{\prime}\right)$ is finite (because $K^{\prime}$ is bounded) and the $F_{y}$ 's are pairwise disjoint, so they define a f.s.r. $T$ with

$$
\begin{gathered}
T^{(0)}=\bigcup_{\Omega^{\prime}} F_{y}, \\
T=\left\{\left(x^{\prime}, x\right) \mid x^{\prime}, x \in F_{y} \quad \text { for some } y \in \Omega^{\prime}\right\} .
\end{gathered}
$$

For each fibre $F_{y}$ of $T$ we have by construction

$$
\sum_{i} \sum_{\mathcal{B} Y} \delta^{-1}(y, x)<\epsilon \sum_{F_{y}} \delta^{-1}(y, x)
$$

where

$$
B_{i}^{y}=\left\{x \in \operatorname{Dom} \phi_{i}, \phi_{i}(x) \in F_{y}, x \notin F_{y}\right\} \cup\left\{x \in \operatorname{Dom} \phi_{i}, \phi_{i}(x) \notin F_{y}, x \in F_{y}\right\} .
$$

Applying lemma 2 to the f.s.r. with fibres

$$
s\left(y \cdot K_{1}\right)=F_{y}^{\prime}, \quad y \in \Omega^{\prime}
$$

we obtain

$$
\sum_{i} \mu\left(B_{i}\right) \leq \varepsilon \mu\left(T^{(0)}\right)
$$

where

$$
B_{i}=\bigcup_{y \in \Omega^{\prime}} B_{i}^{y}
$$

Now $K=\bigcup$ Graph $\phi_{n}$, so éach $\gamma \in K$ is of the form

$$
\gamma=\left(a, \phi_{i}(a)\right)
$$

for some $i$ and $a \in \operatorname{Dom} \phi_{i}$. If

$$
s(\gamma)=\phi_{i}(a) \in T^{(0)},
$$

let $y \in \Omega^{\prime}$ with $\phi_{i}(a) \in F_{y}$. Then, unless $a \in B_{i}^{y}$, one has $r(\gamma) \in F_{y}$ and $\gamma \in T$. In the same way, if $r(\gamma) \in T^{(0)}$, i.e. $a \in F_{y}$ for some $y \in \Omega^{\prime}$, then $\phi_{i}(a) \in F_{y}$ and $\gamma \in T$, unless $a \in B_{i}^{\prime}$. So if

$$
\text { ! } B=\left\{\gamma \in K, \gamma \notin T, s(\gamma) \text { or } r(y) \in T^{(0)}\right\},
$$

then, recalling that $d m(y, x)=d \mu(y)$, one has

\section{Hyperfiniteness}

$$
m(B) \leq \sum_{i} \mu\left(B_{i}\right) \leq \varepsilon \mu\left(T^{(0)}\right)
$$

LEMMA 9. If $R$ is amenable then, for any bounded measurable subset $K$ of $R$ and any $\varepsilon>0$, there exists a f.s.r. $T$ such that

$$
m(K \backslash T)<\varepsilon .
$$

Proof. Let $\mathscr{E}$ be the set of pairs $(T, H)$, where $T$ is a f.s.r. and $H$ a measurable subset of $K$ satisfying the following conditions:

(1) $m(K \backslash H) \leq \varepsilon \mu\left(T^{(0)}\right)$;

(2) $\gamma \in H$ and $s(\gamma)$ or $r(\gamma) \in T^{(0)} \Rightarrow \gamma \in T$. 
For $(T, H),\left(T^{\prime}, H^{\prime}\right) \in \mathscr{E}$, we put $(T, H) \leq\left(T^{\prime}, H^{\prime}\right)$ when

(a) $T^{\prime}$ is an extension of $T$;

(b) $H^{\prime} \subset H, m\left(H \backslash H^{\prime}\right) \leq \varepsilon \mu\left(T^{(0)} \backslash T^{(0)}\right)$.

This defines an ordering on $\mathscr{E}$, and the partially ordered set $\mathscr{E}$ is inductive: for any totally ordered subset $\mathscr{E}^{\prime}$ of $\mathscr{E}$, take a sequence

$$
\left(T_{n}, H_{n}\right) \in \mathscr{E}^{\prime}
$$

with

$$
\operatorname{Sup}_{n} \mu\left(T_{n}^{(0)}\right)=\operatorname{Sup}_{\mathscr{S}^{\prime}} \mu\left(T^{(0)}\right)
$$

and put

$$
T=\bigcup T_{n}, \quad H=\bigcap H_{n} .
$$

One checks (1), (2) for $(T, H)$ so $(T, H) \in \mathscr{E}$ and $(a),(b)$ to see that

$$
\left(T_{n}, H_{n}\right) \leq(T, H) \text { for all } n \text {. }
$$

Thus, unless $\mathscr{E}^{\prime}$ has a largest element, one has

$$
\mathscr{E}^{\prime} \leq(T, H) \text {. }
$$

Now let $(T, H) \in \mathscr{E}$, assume that

$$
\mu\left(T^{(0)}\right) \neq 1
$$

and let us construct

$$
\left(T^{\prime}, H^{\prime}\right) \in \mathscr{E}, \quad\left(T^{\prime}, H^{\prime}\right) \geq(T, H), \quad \mu\left(T^{(0)}\right)>\mu\left(T^{(0)}\right) .
$$

Let

$$
A=\left(T^{(0)}\right)^{\mathrm{c}},
$$

and on $A$ consider the equivalence relation

$$
R_{A}=\{\gamma \in R, s(\gamma) \in A, r(\gamma) \in A\} .
$$

As $\boldsymbol{R}_{A}$ is amenable the Følner condition is satisfied for the bounded subset

$$
H_{A}=\{\gamma \in H, s(\gamma) \in A, r(\gamma) \in A\}
$$

of $H$.

Thus let $T_{1}$ be a f.s.r. such that

$$
\begin{gathered}
T_{1}^{(0)} \subset A, \\
m\left\{\gamma \in H_{A}, s(\gamma) \in T_{1}^{(0)} \text { or } r(\gamma) \in T_{1}^{(0)}, \gamma \notin T_{1}\right\} \leq \varepsilon \mu\left(T_{1}^{(0)}\right) .
\end{gathered}
$$

Put

$$
\begin{gathered}
T^{\prime}=T \cup T_{1}, \\
H \backslash H^{\prime}=\left\{\gamma \in H_{A}, s(\gamma) \text { or } r(\gamma) \in T_{1}^{(0)}, \gamma \notin T_{1}\right\} .
\end{gathered}
$$

Let us check that $\left(T^{\prime}, H^{\prime}\right) \in \mathscr{E}$. We have

$$
\begin{aligned}
& m\left(H \backslash H^{\prime}\right) \leq \varepsilon \mu\left({T^{\prime}(0)}^{(0)}\right), \\
& m\left(K \backslash H^{\prime}\right) \leq m(K \backslash H)+m\left(H \backslash H^{\prime}\right)
\end{aligned}
$$

which gives (1). If $\gamma \in H^{\prime}$ and $s(\gamma) \in T^{\prime(0)}$, then, if $s(\gamma) \in T^{(0)}$, one has $\gamma \in H$ and hence $\gamma \in T$, since $(T, H) \in \mathscr{E}$. If $s(\gamma) \in T_{1}^{(0)}$, then $\gamma \in T_{1} \subset T^{\prime}$. This proves (2). 
To prove $(T, H) \leq\left(T^{\prime}, H^{\prime}\right)$ we have to show that

$$
T=\left\{\gamma \in T^{\prime}, s(\gamma) \text { or } r(\gamma) \in T^{(0)}\right\}
$$

which is clear by construction.

Now using the axiom of choice we have shown that there exists $(T, H) \in \mathscr{E}$ with $T^{(0)}=X$ a.e. Conditions (1), (2) give

(1) $m(K \backslash H) \leq \varepsilon$,

(2) $\gamma \in H \Rightarrow \gamma \in T$ a.e.,

which imply $m(K \backslash T) \leq \varepsilon$.

THEOREM 10. $R$ is hyperfinite if and only if it is amenable.

Proof. If $R$ is hyperfinite it is easy to see that it is amenable.

Conversely, choose a sequence $K_{n}$ of bounded sets with

$$
\bigcup K_{n}=R
$$

and by lemma 9 take f.s.r. $T_{n}$, bounded and such that, for all $n$,

$$
m\left(K_{n} \backslash T_{n}\right)<\varepsilon_{n}, \quad m\left(T_{n} \backslash T_{n+1}\right)<\varepsilon_{n} \quad \text { with } \varepsilon_{n}>0, \quad \sum \varepsilon_{n}<\infty .
$$

Then

$$
\underline{\operatorname{Lim}} T_{n}=R
$$

(where $\underline{\operatorname{Lim}} T_{n}=\bigcup_{n} \bigcap_{k \geq n} T_{k}$ contains $K_{n}$ up to a set of measure $\sum_{n}^{\infty} \varepsilon_{m}$ ). Moreover,

$$
T^{n}=\bigcap_{k \geq n} T_{k}
$$

is a f.s.r.,

$$
T^{n} \subset T^{n+1}
$$

and

$$
\bigcup T^{n}=R \text { a.e. }
$$

\section{Applications}

COROLlARY 11. Let $M$ be an amenable von Neuman algebra and $\mathscr{A}_{1}, \mathscr{A}_{2}$ be Cartan subalgebras of $M$. Then there is an automorphism $\sigma \in$ Aut $M$ such that $\sigma\left(\mathscr{A}_{1}\right)=\mathscr{A}_{2}$. Proof. Let $R_{1}, R_{2}$ be measured countable equivalence relations with cocycles $c_{i}$ such that $\left(\lambda\left(R_{i}\right), \lambda\left(X_{i}\right)\right)$ is isomorphic to $\left(M, \mathscr{A}_{i}\right)$ for $i=1,2$. Then, by proposition $7, R_{i}$ is amenable and hence hyperfinite, so the cocycles $c_{i}$ are trivial. So, by Krieger's theorem [18], let $\sigma$ be an isomorphism of $R_{1}$ on $R_{2}$ preserving the measure class. Then the map which to $\lambda\left(\phi_{1}\right), \phi_{1} \subset R_{1}$, associates $\lambda\left(\sigma\left(\phi_{1}\right)\right)$ extends to an automorphism of $M$ with the required properties (cf. [12]).

COROLlARY 12. Let $R$ be a discrete measured hyperfinite equivalence relation on a measure space $(X, \mu)$, and $\theta$ a non-singular, countable to one, measurable map of $X$ in $X$ such that

$$
x \underset{R}{\widetilde{y}} \text { implies } \theta(x) \underset{R}{\sim} \theta(y) .
$$


Then the equivalence relation $R_{\theta},(y, x) \in R_{\theta}$ if and only if $\exists n, m \in \mathbb{N}$ with $\theta^{n}(x) \underset{R}{\sim} \theta^{m}(y)$, is hyperfinite.

Proof. Let $\left(p_{x}\right)_{x \in X}$ be a measurable family where $p_{x}$ is a state on $l^{\infty}\left(R^{x}\right)$ and $\gamma p_{x}=p_{y}$ for any $\gamma: x \rightarrow y, \gamma \in R$. We construct a measurable family $\left(p_{x}^{\prime}\right)_{x \in X}$, where $p_{x}^{\prime}$ is a state on $l^{\infty}\left(\boldsymbol{R}_{\theta}^{x}\right)$, as follows. Let $F$ be an element of $l^{\infty}\left(\boldsymbol{R}_{\theta}^{x}\right)$. Then, since for each $k \in \mathbb{N}$ the $R$-equivalence class of $\theta^{k}(x)$ is contained in the $R_{\theta}$ class of $x$, we can restrict $F$ to $R^{\theta^{k(x)}}$ and obtain the scalar

$$
p_{\theta^{k}(x)}(F(\cdot))=\alpha_{k} .
$$

Now fixing an invariant mean $\rho$ on $l^{\infty}(\mathbb{N})$, we set

$$
p_{x}^{\prime}(F)=\rho(\alpha)
$$

If we replace $x$ by $x^{\prime} \underset{R}{\sim} x$, we have

$$
\theta^{k}\left(x^{\prime}\right) \underset{R}{\sim} \theta^{k}(x)
$$

so that none of the scalars $\alpha_{k}$ changes, and

$$
p_{x^{\prime}}^{\prime}(F)=p_{x}^{\prime}(F) \text {. }
$$

If we replace $x$ by $\theta^{k}(x)$, the sequence $\alpha$ is shifted, and by the invariance of $\rho$ we obtain

$$
p_{\theta^{k}(x)}^{\prime}(F)=p_{x}^{\prime}(F)
$$

This shows that, if

$$
x \underset{R_{\theta}}{\sim} x^{\prime}
$$

then

$$
p_{x}^{\prime}(F)=p_{x^{\prime}}^{\prime}(F),
$$

i.e. $p^{\prime}$ is invariant. The only delicate point is that one needs to choose $\rho$ in such a way that $p^{\prime}$ be measurable. There are two ways out: the first is to use a general result of G. Mokobodski [20], asserting the existence of means like $\rho$ which are universally measurable. The second is to translate the above formulae algebraically in terms of the projection $P$, and to define $P^{\prime}(F)$ for $F \in L^{\infty}\left(R_{\theta}\right)$ using a weak limit in $L^{\infty}(X, \mu)$.

We shall leave the tedious verification to the reader. The main result of [7] is the special case of the above corollary 12 when $\theta$ is an invertible element of the normalizer of the equivalence relation $R$. The following theorem, stated by Veršik in [29], is obtained as a corollary:

COROLLARY 13. Let $T$ be a non-singular endomorphism of $(X, \mu)$ such that $T^{-1}\{x\}$ is countable for a.e. $x \in X$. Then the equivalence relation, $x \sim y$ if and only if $\exists n, m \in \mathbb{N}$ such that $T^{n} x=T^{m} y$, is hyperfinite.

Proof. Apply corollary 12 with $R=\{(x, x), x \in X\}$ and $T$ in place of $\theta$. Note, however, the simpler form of $p_{x}^{\prime}$ in this special case.

In [2] R. Bowen proved the hyperfiniteness of Anosov foliations by first establishing an orbit equivalence with a certain endomorphism $T$ and then showing by direct 
computations the hyperfiniteness. Corollary 13 shows that the special form of $T$ plays no role. Also, both Bowen and Veršik deduce from this that the action of $\mathrm{SL}(2, \mathbb{Z})$ on $P_{1}(\mathbb{R})$ is hyperfinite. We obtain the result from the very general:

COROLlARY 14. Let $G$ be a locally compact group, $P$ an amenable closed subgroup and $\Gamma$ a discrete subgroup of $G$. Then the action of $\Gamma$ on $G / P$ is hyperfinite.

Proof. By [31] this action is amenable.

We shall end this paper with a brief discussion of the case of equivalence relations with not necessarily countable orbits. Let $X$ be a standard Borel space and $R \subset X \times X$ a Borel subset which is an equivalence relation. The hypothesis of discreteness of $R$ is now replaced by the hypothesis of existence of a transverse function $\nu$ for $R$ with $\nu^{x} \neq 0, \forall x \in X$ (cf. [4]). For each $x, \nu^{x}$ is a $\sigma$-finite measure on $R^{x}$ and one assumes:

(1) that $\nu$ is invariant: i.e. $\gamma \nu^{x}=\nu^{y}, \forall \gamma: x \rightarrow y, \gamma \in R$;

(2) that $\nu$ is proper: i.e. $R$ is a countable union of Borel sets $A_{n}$ such that $\nu^{x}\left(A_{n}\right)$ is bounded for each $n \in \mathbb{N}$.

Fixing such a transverse function $\nu$, and a module $\delta$, i.e. a Borel homomorphism of the groupoid $R$ to $\mathbb{R}_{+}^{*}$, the transverse measures $\Lambda$ of module $\delta$ on $R$ correspond bijectively to the ordinary measures $\mu$ on $X$ such that the conditional measure of $\mu$ on any $R$-equivalence class $l$ is proportional to $\delta^{-1} \nu$ (cf. [4] thm. 3, § II) or, equivalently, such that the measure

on $R$ satisfies

$$
m=\int \nu^{x} d \mu(x)
$$

$$
m^{-1}=\delta^{-1} m
$$

The advantage of transverse measures is that we can, at will, change our choice of $\nu$ to another $\nu^{\prime}$, where $\nu^{\prime x}$ can be singular relative to $\nu^{x}$ for any $x \in X$. Retaining from $\Lambda$ only its class, i.e. the notion of saturated $\Lambda$ negligible subsets of $X$ (cf. [4] def. 7), we shall now define the amenability of the system $(R$, class of $\Lambda)$. We shall first define it relative to a choice of $\nu$ and then show that this choice is unimportant. Fixing $\nu$, the amenability means the existence for a.e. $x \in X$ of a state $p_{x}$ on $L^{\infty}\left(R^{x}, \nu^{x}\right)$ in such a way that

(1) $\gamma p_{x}=p_{y}, \forall \gamma: x \rightarrow y, \gamma \in R$;

(2) for any $f \in L^{\infty}(R, m)\left(m=\int \nu^{x} d \mu\right)$, the function on $X$, given by $x \mapsto p_{x}(f)$, is $\mu$-measurable.

LEMMA 15. The amenability of $(R$, class of $\Lambda)$ is independent of the choice of $\nu$.

Proof. Let $\nu^{\prime}$ be another transverse function on $R$ with $\nu^{\prime x} \neq 0$ a.e. By [8] prop. 6, $\S 1$, there exists a measurable map $x \rightarrow \lambda^{x}$ from $X$ to positive measures on $R$, with $\lambda^{x}$ carried by $R^{x}$ for any $x \in X$ and such that

$$
\int f(z) d \nu^{\prime x}(x, z)=\iint f(z) d \lambda^{y}(y, z) d \nu^{x}(x, y)
$$

for any positive measurable $f$ on $\{z, z \sim x(R)\}$. Note that by the invariance of $\nu$ (resp. $\left.\nu^{\prime}\right), d \nu^{x}(x, z)$ just depends on $z$ (resp. $d \nu^{\prime x}$ ), and defines a measure $\nu^{l}$ on the 
$R$-equivalence class of $x$. The above equality shows the existence of a bounded positive map $U_{l}$ of $L^{\infty}\left(l, \nu^{l}\right)$ in $L^{\infty}\left(l, \nu^{\prime}\right)$, with $U_{l}(1)=1$, defined as the transpose of the map from $L^{1}\left(l, \nu^{\prime}\right)$ in $L^{1}\left(l, \nu^{l}\right)$ to which $f$ associates the function

$$
y \in l \rightarrow \int f(z) d \lambda^{y}(y, z) .
$$

Then if $\left(p_{l}^{\prime}\right)_{l \in \Omega}, \Omega$ the space of $R$ equivalence classes, is a measurable family of states on $L^{\infty}\left(l, \nu^{\prime l}\right)$, the family

$$
p_{l}=p_{i}^{\prime} \circ U_{l}
$$

is a measurable family of states on $L^{\infty}\left(l, \nu^{l}\right)$.

Recall that, given an equivalence relation $R$ as above, a transversal $T$ is a measurable subset of $X$ such that the restriction of $R$ to $T$ is discrete. By a recent result of A. Ramsay [25], the existence of a transverse function $\nu$ with $\nu^{x} \neq 0, \forall x \in X$, implies the existence of a transversal $T$ which intersects almost all $R$-equivalence classes. $T$ gives rise to a transverse function $\nu_{T}$, by defining $\nu_{T}^{x}$ to be the measure supported on $\{y \in T: y \sim x\}$ with

$$
\nu_{T}(\{y\})=1 .
$$

The measure $\mu$ on $X$ corresponding to any transverse measure $\Lambda$ in our class clearly has its support in $T$, and setting

$$
R_{T}=R \cap(T \times T),
$$

we obtain a d.m.e.r. on $(T, \mu)$. This we call the restriction of $(R, \Lambda)$ to $T$. Changing to a $\Lambda^{\prime}$ in the same class means changing to a $\mu^{\prime}$ in the same class, so we may speak of the restriction of $(R$, class of $\Lambda)$ to $T$. The amenability of $(R$, class of $\Lambda)$ amounts precisely to the existence of an invariant family $\left(p_{x}\right)$ on the equivalence classes of $R_{T}$, by lemma 15 . Thus by theorem 10 we obtain:

COROLlary 16. Let $(R$, class of $\Lambda)$ be an equivalence relation with transverse measure class. Then it is amenable if and only if its restriction to some (equivalently any) transversal is generated by a single transformation.

Remark. Instead of using transverse measures on equivalence relations, one can, of course, use Mackey's notion of virtual group. Corollary 16 can be restated as:

COROLlary 17. Any principal amenable virtual group is similar to a virtual subgroup of the group of integers.

COROLlary 18. Let the amenable locally compact Polish group $G$ act ergodically and non-singularly on the standard space $(X, \mu)$. Then the ergodic equivalence relation obtained from the orbits of $G$ is hyperfinite.

Remark. For discrete $G$ and free actions, this is obtained in Ornstein-Weiss [24].

Proof. Let $\rho$ be a right invariant mean on $L^{\infty}(G, d g)$, obtained as a medial limit from a Følner sequence in $G$. For each $x \in X$, let $p_{x}$ be the state on $L^{\infty}$ (orbit of $x$ ) defined by

$$
p_{x}(f)=\rho(\tilde{f})
$$


where $\tilde{f}(g)=f(g x), \forall g \in G$. By [20] the family $\left(p_{x}\right)$ is measurable and, by the invariance of $\rho$,

$$
p_{g x}=p_{x}
$$

for any $g \in G$. So corollary 16 completes the proof.

We obtain from this the following generalization of (part of) the Ambrose theorem on flows, answering a question raised by Mackey [19]:

COROLlary 19. Any ergodic non-singular free action of an amenable locally compact Polish group $G$ is the range of a transient homomorphism of an integer action.

This follows from Feldman-Moore-Hahn [13], cor. 7.9. We note that this has been shown for connected nilpotent groups by R. Zimmer [30], and for solvable groups by A. Stëpin (personal communication).

The next proposition is of particular interest when applied with corollary 16 to foliations with a fixed Riemannian structure $g$ along the leaves, the transverse function $\nu$ being given by the Riemannian volume element, and the process $\pi$ defining the usual harmonic functions along the leaves.

Proposition 20. Let $(R$, class of $\Lambda)$ be as above, $\nu$ a transverse function with $\nu^{x} \neq 0$ a.e., and $\pi$ a Markov process on $X$, with $\pi^{x}$ carried by the $R$-equivalence class of $x$ for each $x \in X$, which is non-singular relative to $\nu$ in the following sense. For each $R$-equivalence class the measure

$$
\int \pi^{x} d \nu^{l}(x)
$$

on l should be absolutely continuous with respect to $\nu^{\prime}$. Then if the only bounded $\nu$-measurable $\pi$-harmonic functions on each $R$-equivalence class $l$ are the constants, the equivalence relation $(R$, class of $\Lambda)$ is amenable.

Proof. For each $R$-equivalence class $l \in \Omega$, the equality

$$
\left(U_{l} f\right)(x)=\int f(z) d \pi^{x}(z)
$$

defines a bounded positive map $U_{l}$ of $L^{\infty}\left(l, \nu^{l}\right)$ into itself such that

$$
U_{l}(1)=1 \text {. }
$$

Now any weak limit in $L^{\infty}\left(l, \nu^{l}\right)$ of the sequence

$$
\frac{1}{m} \sum_{1}^{m}\left(U_{l}\right)^{k} f
$$

is $\pi$-harmonic, and hence by hypothesis is equal to a constant. Choosing once and for all a measurable limit procedure [20], one obtains a measurable family $\left(p_{l}\right)_{l \in \Omega}$ of states on $L^{\infty}\left(l, \nu^{l}\right)$.

COROLLARY 21. Let $V$ be a manifold, $F \subset T V$ a sub-bundle defining a foliation of $V$,\| $\|$ a Euclidean metric on $F$. Assume that each leaf of this foliation is of polynomial growth (for the Riemannian metric defined by \|\| ), then the equivalence relation, 
$x \sim y$ in $V$ if and only if the leaf of $x$ is the leaf of $y$, with the smooth measure class, is hyperfinite.

Proof. For a fixed leaf $M$, let $x \in M$ and $B_{n}(x)$ be the ball of radius $n$ and centre $x$. Define a state $p_{x}$ on $L^{\infty}(M)$ by

$$
p_{x}(f)=\text { Medial Limit } u_{n},
$$

where

$$
u_{n}=\frac{1}{\text { volume }\left(B_{n}(x)\right)} \int_{B_{n}(x)} f(y) d y .
$$

The polynomial growth of $M$, shows that $p_{x}$ is independent of the choice of $x$ in $M$, hence the amenability of this equivalence relation follows, and corollary 16 completes the proof.

J. F. was partly supported by NSF grant MCS 78-06718.

\section{REFERENCES}

[1] R. M. Belinskaya. Partitioning of a Lebesgue space into trajectories which are definable by ergodic automorphisms. Funkcional Anal. i. Prilozen 2 (1968), 4-16 (in Russian).

[2] R. Bowen. Anosov foliations are hyperfinite. Ann. Math. 106 (1977), 549-565.

[3] R. V. Chacon \& N. Friedman. Approximation and invariant measures. Z. Wahrscheinlichkeitstheorie verw. Geb. 3 (1965), 286-295.

[4] A. Connes. Sur la théorie non commutative de l'intégration. Lecture Notes in Math. No. 725, pp. 19-143. Springer-Verlag: Berlin, 1979.

[5] A. Connes. Classification of injective factors. Annals of Math. 104 (1976), 73-115.

[6] A. Connes. On the cohomology of operator algebras. J. Funct. Analysis 28(2) (1978), 248-253.

[7] A. Connes \& W. Krieger. Measure space automorphisms, the normalizer of their full groups and approximate finiteness. J. Funct. Analysis 29 (1977), 336.

[8] J. P. Conze. Entropie d'un groupe abélien de transformations. $Z$. Wahrscheinlichkeitstheorie verw. Geb. 25 (1972), 11-30.

[9] H. Dye. On groups of measure preserving transformations. I. Amer. J. Math. 81 (1959), 119-159.

[10] H. Dye. On groups of measure preserving transformations. II. Amer. J. Math. 85 (1963), 551-576.

[11] J. Feldman \& D. Lind. Hyperfiniteness and the Halmos-Rohlin theorem for non-singular actions. Proc. A.M.S. 55 (1976), 339-344.

[12] J. Feldman \& C. Moore. Ergodic equivalence relations, von Neumann algebras and cohomology. I and II. Trans. A.M.S. 234 (1977), 289-324.

[13] J. Feldman, C. Moore \& P. Hahn. Orbit structure and countable sections for actions of continuous groups. Adv. Math. 28 (1978), 186-230.

[14] V. Ya. Golodets. Crossed products of von Neumann algebras. Uspekhi Math. Nauk. 26(5) (1971), 3-50.

[15] E. Hopf. Theory of measure and invariant integrals. Trans. A.M.S. 39 (1932), 373-393.

[16] A. Ionescu Tulcea. On the category of certain classes of transformations in ergodic theory. Trans. A.M.S. 114 (1965), 261-279.

[17] Y. Katznelson \& B. Weiss. Commuting measure preserving transformations. Israel J. Math. 12 (1972), 161-173.

[18] W. Krieger. On ergodic flows and the isomorphism of factors. Math. Ann. 223 (1976), 19-70.

[19] G. W. Mackey. Ergodic theory and virtual groups. Math. Ann. 166 (1966), 187-207.

[20] G. Mokobodski. Limites médiales. Exposé de P. A. Meyer. Sem. Probabilités, Université de Strasbourg. 1971-72. Lecture Notes in Math. No. 321, pp. 198-204, Springer-Verlag: Berlin, 1973.

[21] F. J. Murray \& J. von Neumann. On rings of operators. Ann. Math. 37 (1936), 116-229.

[22] F. J. Murray \& J. von Neumann. Rings of operators IV. Ann. Math. 44 (1943), 716-804.

[23] D. Ornstein \& B. Weiss. The Kakutani-Rohlin theorem for solvable groups. (Preprint, 1976). 
[24] D. Ornstein \& B. Weiss. Ergodic theory of amenable group actions I. The Rohlin lemma. Bull. A.M.S. 2 (1980), 161.

[25] A. Ramsay. Measured groupoids and countable equivalence relations. Advances in Math. (to appear).

[26] J. Schwartz. Two finite, non hyperfinite, non isomorphic factors. Comm. Pure. Appl. Math. 16 (1963), 19-26.

[27] C. Series. The Rohlin tower theorem and hyperfiniteness for actions of continuous groups. Israel J. Math. 30 (1978), 99-122.

[28] A. M. Veršik. Non measurable decompositions, orbit theory, algebras of operators. Dokl. Acad. Nauk. SSSR 199 (1971), 1004-1007.

[29] A. M. Veršik. The action of PSL $(2, \mathbb{Z})$ on $\mathbb{R}^{1}$ is approximable. Uspekhi Matematicheski Nauk. 33(1) (1978), 209-210 (in Russian).

[30] R. Zimmer. Hyperfinite factors and amenable ergodic actions. Inv. Math. 41 (1977), 23-31.

[31] R. Zimmer. Cocycles and the structure of ergodic group actions. Israel J. Math. 26 (1977), 214-220.

[32] R. Zimmer. Amenable ergodic group actions and an application to Poisson boundaries of random walks. J. Funct. Analysis 27 (1978), 350-372. 